\title{
COMPORTAMIENTO PRODUCTIVO DE HÍBRIDOS DE PAPAYA (Carica papaya L.) EN GUÁPILES, COSTA RICA.
}

\author{
Antonio Bogantes Arias', Eric Mora Newcomer ${ }^{2}$ \\ RESÚMEN
}

Comportamiento productivo de híbridos de papaya (Carica papaya L.) en Guápiles, Costa Rica. Este experimento se realizó en Guápiles, Limón, Costa Rica durante los años 2008 y 2009. El objetivo fue determinar la productividad de nuevos híbridos de papaya. Los híbridos H-10 G (MH03-7 x T2); H-11 G (MH03-7 x HC95-8); H-10 P (MH03-10 x T2); H-11 P (MH03-10 $x$ HC95-8) y H Pococí (testigo), se sembraron en un diseño de bloques completos al azar con cuatro repeticiones. Se evaluó altura de planta y de inserción de la primera fruta, grosor del tallo, número y peso de frutos, firmeza, sólidos solubles (brix) y severidad de antracnosis (Colletotrichum gloesporioides) en la fruta, así como la incidencia de carpelodia en flores hermafroditas. Las plantas del híbrido $\mathrm{H}-10 \mathrm{P}$ fueron $48 \mathrm{~cm}$ más altas que las del híbrido más bajo. Los híbridos derivados de la línea MHO3-10 produjeron 12,7 kg más de fruta por planta con respecto a los derivados de la línea MHO3-7. En general, el rendimiento en todos los híbridos fue superior a $40 \mathrm{~kg}$ por planta. La firmeza de cáscara y pulpa en la fruta varió entre 70 y 136 newton (N). Los híbridos de la línea MHO3-10, superaron en 1,02 grados brix a los híbridos con MHO3-7. El porcentaje de severidad de antracnosis en fruta estuvo por debajo de seis. Se observaron flores con carpelodia en plantas de $\mathrm{H}-10 \mathrm{G}$ y H-11G.

Palabras clave: rendimiento, firmeza, grados brix, antracnosis.

\section{INTRODUCCIÓN}

En las localidades de Guácimo, Pococí y alrededores (región Huetar Atlántica) así como, en la Fortuna y en el Tanque de San Carlos (región Huetar Norte) de Costa Rica, se produce cerca del $50 \%$ de la producción de papaya. Se requiere desarrollar nuevos materiales con potencial comercial y de adaptación a las condiciones de estas zonas en las que la precipitación anual es superior a los $3000 \mathrm{~mm}$ y la temperatura promedio supera los $24{ }^{\circ} \mathrm{C}$ (Mora y Bogantes 2004 a).

El mejoramiento genético en papaya es una opción para obtener materiales adaptados y con las características apropiadas a las zonas productoras (Chan 1992, Loyola y Firmino 2001, Goenaga et al. 2001, Mora y Bogantes 2004 a, b; David et al. 2006, Alonso etal. 2008 a y Alonso et al. 2009 a). La diversidad genética en los materiales silvestres y cultivados de papaya, permite hacer prospección y selección de accesiones locales que junto a la introducción de nuevos genotipos foráneos, constituyen dos vías fundamentales para aumentar la variabilidad (Alonso et al. 2009 b).

En Costa Rica se ha logrado la producción de nuevos híbridos con el cruzamiento de variedades locales e introducidas, se aprovecha la heterosis, se mejora el sabor y se obtienen altos grados brix en la fruta (Mora y Bogantes 1999-2002). Esta misma estrategia se sigue en otros países, como México y Cuba con la introducción o el uso de materiales de altos grados brix como la Maradol, Sunrise y Sunset (grupo Solo) o la Red Lady (Rivas et al. 2003; Alcántara et al. 2010, Alonso et al. 2008 b).

Algunas características de producción importantes para la selección adecuada de variedades de papaya son la altura de la planta, el número de frutas, la altura de la primera fruta y el peso de la misma (Loyola y Firmino 2001).

\footnotetext{
${ }^{1}$ Instituto Nacional de Innovación y Transferencia en Tecnología Agropecuaria, INTA. Costa Rica. abogantes@inta.go.cr.

${ }^{2}$ Estación Experimental Fabio Baudrit Moreno, UCR, Costa Rica. emoran@racsa.co.cr.
} 
También, son importantes las características de calidad del fruto, como grados brix, color rojo y firmeza en la pulpa así como la tolerancia a Colletotrichum gloesporioides que afecta la fruta, principalmente en la etapa de poscosecha (Solano y Arauz 1995).

Se han documentado pérdidas entre el 25 y 50 $\%$ en frutos de papaya cosechada, causadas por la antracnosis (Páez s.f.). En Costa Rica es la enfermedad que más afecta a la fruta que se exporta a Canadá. Barrientos (2010) ${ }^{3}$, indica que un $75 \%$ del rechazo de la fruta exportada a ese país, por el Centro Agrícola Cantonal de Guácimo durante el año 2010, fue por este patógeno. Es evidente que la resistencia es una característica importante en los procesos de selección (Mora y Bogantes 1999-2002).

Las variedades criollas (multilíneas) son aún empleadas por los productores en Costa Rica (Guzmán 1998). Éstas tienen deficiencias en estabilidad productiva y calidad gustativa de la fruta, lo cual, ha repercutido negativamente en el consumo de la papaya. La liberación del híbrido Pococí en el 2006 introdujo ventajas relacionadas con el rendimiento, la calidad de la fruta, y la estabilidad o uniformidad entre las plantas. Este híbrido, produce una fruta con un peso promedio de 1,3 kg (Bogantes y Mora 2006); su rendimiento así como el peso y el aroma le han agradado al productor y al consumidor.

En la actualidad, existen otros usuarios, miembros de la agrocadena de papaya, como los industriales y los hoteleros que requieren de una fruta con dos kilogramos de peso, uniforme, sana y con un brix alto (no menos de 10 grados). Además, se deben considerar otras oportunidades o nichos de mercado como el de fruta pequeña (entre 600 y $800 \mathrm{~g}$ ), con poca cavidad interna, similar al tipo Solo (Alonso et al. 2008 b), especial para exportar a Europa y Estados Unidos.

Esta investigación tuvo como objetivo, determinar la productividad de nuevos híbridos de papaya, en Guápiles, Costa Rica.

\section{MATERIALES Y MÉTODOS}

El experimento se inició en setiembre del 2008 y finalizó en noviembre del 2009, en la finca de un productor de papaya, localizado en el distrito de La Rita, cantón de Pococí, provincia de Limón. La altura aproximada de dicha región es de $225 \mathrm{msnm}$, con una temperatura promedio de $24,6^{\circ} \mathrm{C}$ y una precipitación anual promedio de $4380 \mathrm{~mm}$. Los tratamientos fueron híbridos producidos en el proyecto conjunto entre la Universidad de Costa Rica (UCR) y el Instituto Nacional de Innovación y Transferencia en Tecnología Agropecuaria (INTA-Costa Rica) y se describen en el Cuadro 1.

Las líneas progenitoras tienen diferentes orígenes:

a. Lalínea T2 es un material criollo seleccionado y autopolinizado durante dos años.

Las líneas MH03-7, MH03-10 y HC95-8 tienen tres y seis generaciones de autofecundación respectivamente y fueron obtenidas de una población segregante a partir de material autóctono hibridizado con variedades de Cuba y Hawaii (Mora y Bogantes 1999-2002).

Se utilizó como testigo el híbrido Pococí liberado en el 2006 (Bogantes y Mora 2006).

Cuadro 1. Híbridos de papaya y sus respectivos progenitores. Guápiles, Limón,CR. 2010.

\begin{tabular}{|l|l|}
\hline Tratamientos & Líneas progenitoras \\
\hline 1. Hibrido H-10 G & MH03-7 x T2 \\
\hline 2. Híbrido H-11 G & MH03-7 x HC95-8 \\
\hline 3. Hibrido H-10 P & MH03-10 x T2 \\
\hline 4. Híbrido H-11 P & MH03-10 x HC95-8 \\
\hline 5. Hibrido Pococí & Testigo absoluto \\
\hline
\end{tabular}

El diseño experimental consistió en un bloques completos al azar con cuatro repeticiones. La parcela total para cada repetición, estuvo constituida por un surco de 6 plantas

\footnotetext{
${ }^{3}$ Barrientos, M. 2010. Antracnosis (Colletotrichum gloeosporioides)en papaya para exportación. Centro Agrícola Cantonal de Guácimo. Guácimo, Pococí. Comunicación personal.
} 
sembradas a 2,5 m entre sí y 2,5 m entre surco. Se aplicó el manejo del productor que consistió en fertilizar con la fórmula comercial 10-3010 (30-60 g planta - $\left.{ }^{1}\right)$ a la siembra. Después de la siembra se aplicó 15-3-31 y 18-5-156-1.2 (50-100 g planta -1) en forma alterna y mensual desde los tres hasta los 14 meses. Lo anterior se complementó con la aplicación de microlementos foliares cada mes. El combate de Colletotrichum gloesporioides en la fruta se efectuó con aplicaciones preventivas de benomil y mancozeb (2 g p.c. litro $\left.{ }^{-1}\right)$ Para el combate de Toxotripana curvicauda se usó cipermetrina (1 cc p.c. litro $-^{1}$ ) y el control de malezas se efectuó con glifosato (90-100 cc p.c. litro ${ }^{-1}$ ).

\section{Variables evaluadas}

Altura total de la planta $(\mathrm{cm})$. Se midió desde la base hasta el ápice terminal, a los ocho meses de edad.

Grosor del tallo (cm). Se midió a $50 \mathrm{~cm}$ del suelo, a los ocho meses de edad.

Altura de la primera fruta (cm). Se midió desde el suelo hasta la inserción de la primera fruta, a los siete meses de edad.

Número y peso de frutos (g). Se contó el número de frutos de todo el primer flujo de producción (cinco meses) y se tomó el peso del fruto. Adicionalmente, se contó el número de plantas con al menos una flor hermafrodita con carpelodia. Todo a los ocho meses de edad.

Sólidos solubles (grados Brix) de la pulpa. Se evaluó en fruta $100 \%$ madura (amarilla externamente) de plantas con 10 meses de edad. (Mora y Bogantes 2004 b).

Firmeza (newton) de pulpa y cáscara. Se midió con un penetrómetro manual, en el centro de la fruta $100 \%$ madura de plantas con 10 meses de edad.

Porcentaje de Severidad de antracnosis (Colletotrichum gloesporioides) en fruta.
Se calculó haciendo una observación visual del porcentaje de área con síntomas en la fruta $100 \%$ madura de plantas con 10 meses de edad, según la escala de severidad de Solano y Arauz (1995). La medición de ésta variable se basó en el inóculo natural de la plantación, proveniente de peciolos, hojas, flores y frutas (Durán et al. 2000).

La muestra para el número de frutos por planta, altura de la planta, altura de la primera fruta y grosor del tallo, consistió de dos plantas por repetición para un total de ocho por híbrido o tratamiento. La muestra para peso de fruta por planta, porcentaje de antracnosis, firmeza de la pulpa y brix consistió en dos frutas por planta, cuatro por repetición para un total de 16 frutas por tratamiento.

El brix se midió con un refractómetro de mano graduado de 0 a $32 \%$. La firmeza se midió con un penetrómetro de 200 newton $(\mathrm{N})$, con un extremo (accesorio) plano y redondo de $1 \mathrm{~cm}$ de diámetro.

Los datos finales fueron sometidos a pruebas de normalidad (Shapiro-Wilk), homogeneidad de varianzas y el análisis de varianza. Motivados por las características de las líneas progenitoras (Cuadro 1) se hicieron comparaciones por análisis de contrastes entre los híbridos con MHO3-7 (H-10G y $\mathrm{H}-11 \mathrm{G})$ y los híbridos con MHO3-10 (H-10P y $\mathrm{H}-11 \mathrm{P})$. También, entre los híbridos con T2 (H-10G y H-10P) y los híbridos con HC95-8 (H-11G y H-11P) así como el híbrido Pococí con los cuatro híbridos restantes. Todos los análisis fueron realizados utilizando el sistema estadístico SAS (López y López 1995).

\section{RESULTADOS Y DISCUSIÓN}

\section{Altura de planta, fruta y grosor del tallo}

La altura total de las plantas y el grosor del tallo fue diferente entre híbridos $(p=0,0001 \mathrm{y}$ 0,006 respectivamente). A los ocho meses de edad la diferencia promedio entre la altura de las plantas más altas $(\mathrm{H}-10 \mathrm{P})$ y las más bajas $(\mathrm{H}-10 \mathrm{G})$ fue de $48 \mathrm{~cm}$ (Cuadro 2). 
Cuadro 2. Altura de planta, grosor del tallo y altura desde el suelo a primera fruta de híbridos de papaya a los ocho meses después de la siembra. Guápiles, Limón, CR. 2009.

\begin{tabular}{|c|c|c|c|}
\hline Tratamiento & Altura planta $(\mathrm{cm})$ & Grosor tallo $(\mathrm{cm})$ & Altura primera fruta $(\mathrm{cm})$ \\
\hline $\mathrm{H}-10 \mathrm{P}$ & 205 & 9,58 & 73,38 \\
\hline $\mathrm{H}-11 \mathrm{P}$ & 199 & 8,50 & 78,12 \\
\hline $\mathrm{H}-$ Pococí & 185 & 9,84 & 76,75 \\
\hline $\mathrm{H}-11 \mathrm{G}$ & 172 & 8,62 & 68,00 \\
\hline $\mathrm{H}-10 \mathrm{G}$ & 157 & 8,04 & 73,50 \\
\hline
\end{tabular}

Los $\mathrm{H}-10 \mathrm{P}$ y H-11P que comparten a la línea MHO3-10, mostraron mayor altura (promedio de $38 \mathrm{~cm}$ ) con respecto a los derivados de la línea MHO3-7 (H-10G y H-11G). (Cuadros 2 y 3). Las plantas de la línea progenitora MHO3-10 fueron más altas y de entrenudos más largos que los de la línea MHO3-7.

Las líneas T2 y HC95-8, son parecidas en altura y tuvieron un efecto similar en el crecimiento de sus respectivos híbridos (H-10G, H-10P y H-11G, H-11P). La altura del híbrido Pococí usado como testigo, fue igual al promedio de los cuatro híbridos restantes. Con respecto al grosor del tallo, las plantas del Híbrido Pococí fueron $1,15 \mathrm{~cm}$ más gruesas que el promedio de los otros cuatro híbridos (Cuadros 2 y 3 ).

Cuadro 3. Estimados de las diferencias de altura de planta y grosor del tallo entre grupos de híbridos de papaya, ocho meses después de la siembra. Guápiles, Limón, CR. 2009.

\begin{tabular}{|c|c|c|}
\hline Tratamientos (híbridos) & Altura planta $(\mathrm{cm})$ & Grosor tallo $(\mathrm{cm})$ \\
\hline $\begin{array}{c}\mathrm{H}-10 \mathrm{G} \text { y } \mathrm{H}-11 \mathrm{G}\left(\mathrm{MHO}-7^{*}\right) \text { vs } \\
\mathrm{H}-10 \mathrm{P} \text { y } \mathrm{H}-11 \mathrm{P}\left(\mathrm{MHO}-10^{*}\right)\end{array}$ & $\begin{array}{c}-37.75 \\
(p=0,0001)\end{array}$ & $\begin{array}{c}-0,71 \\
(p=0,07 \text { n.s. })\end{array}$ \\
\hline $\begin{array}{l}\mathrm{H}-10 \mathrm{G} \text { y } \mathrm{H}-10 \mathrm{P}\left(\mathrm{T}^{*}\right) \text { vs } \\
\mathrm{H}-11 \mathrm{G} \text { y } \mathrm{H}-11 \mathrm{P}\left(\mathrm{HC} 95-8^{*}\right)\end{array}$ & $\begin{array}{c}-4,50 \\
(p=0,44 \text { n. s. })\end{array}$ & $\begin{array}{c}0,25 \\
(p=0,53 \text { n. s.) }\end{array}$ \\
\hline Todos vs H. Pococí & $\begin{array}{c}-1,75 \\
(p=0,85 \text { n. s. })\end{array}$ & $\begin{array}{l}-1.15 \\
(p=0,01)\end{array}$ \\
\hline
\end{tabular}

* Líneas progenitoras

La altura de las plantas en los híbridos, con excepción del híbrido $\mathrm{H}-10 \mathrm{G}$, es similar a la de otros híbridos como Tainung 1 y Scarlett Princess, con un año de edad (Alonso et al. 2009 a), y a la de las variedades Red Lady y Sunset Solo en México, con 1,88 y 1,81 m (Rivas et al. 2003). En aquellos genotipos sin problemas de esterilidad femenina, la planta alta (con dos metros), es una característica importante porque podría estar relacionada con una mayor cantidad de frutas. Sin embargo, a mayor altura de la planta más dificultad para cosechar la fruta (Alonso et al. 2009 a). En nuestro medio se han observado plantas muy bajas (con menos de $1,50 \mathrm{~m}$ ) y productivas pero con problemas de compresión de frutas y con entrenudos muy cortos, que reducen su ciclo productivo.

La inserción de la primera fruta en la planta fue muy similar entre los híbridos $(p=0,39)$, estuvo a una altura entre 68 y $78 \mathrm{~cm}$ del suelo (Cuadro 2). Alonso et al. (2008 a), reportan una correlación positiva entre la altura de la planta y la primera fruta (flor) en tres cultivares del grupo Solo evaluados en Cuba. En México, Rivas et al. (2003) reportan la altura de la primera fruta en las variedades Sunrise y Sunset Solo a 88 y $90 \mathrm{~cm}$ del suelo. El inicio de la producción con fruta a poca 
altura es considerada como una característica importante en papaya, tanto es así que Alonso et al. (2009 a), resaltan cultivares con primera fruta a una altura entre 45 y $50 \mathrm{~cm}$ del suelo y destacan como una desventaja en la Tainung 1 , la producción de la primera fruta a los $86 \mathrm{~cm}$. En México, Alcántara et al. (2010), reportan una altura de inserción de la primera fruta desde los $64 \mathrm{~cm}$ del suelo en papaya Maradol y hasta 77 y $108 \mathrm{~cm}$ en otros cultivares criollos e introducidos. Para condiciones de precipitación por encima de los $4000 \mathrm{~mm}$, típicas de la región Huetar Atlántica de Costa Rica, la altura de la primera fruta debería ser mayor a $80 \mathrm{~cm}$, para disminuir la incidencia de Phytophtora $s p$ que es favorecido por el salpique del agua en la fruta. Después de las lluvias queda suelo proveniente del salpique en las frutas y en el tallo, en los primeros $50 \mathrm{~cm}$ de altura (Mora y Bogantes (2004 a). Por otra parte, una excesiva precocidad de la planta con fruta muy baja, puede estar asociada a una vida útil menor de la plantación (Mora y Bogantes 1999-2002).

\section{Número y peso de frutas}

El número de frutos por planta entre los híbridos varió entre 30,50 y 51,12 ( $p=0,01)$ (Cuadro 4). En México el híbrido 'Azteca' en etapa de prueba, produjo un promedio de 38,5 frutos por planta, comparado con 33,9 en la variedad 'Maradol' (Mirafuentes y Azpeitia, 2008). En Cuba Alonso et al. (2009 a), obtuvieron 59, 50 y 40 frutos por planta en los híbridos Tainung 01, Scarllet Princess y la variedad Maradol, respectivamente.

Cuadro 4. Número y peso promedio de frutas, para los primeros cinco meses de cosecha, en cinco híbridos de papaya. Guápiles, Limón, CR. 2009

\begin{tabular}{|l|c|c|c|}
\hline Tratamiento & Número frutos & Peso fruto $(\mathrm{g})$ & Peso por planta $(\mathrm{kg})$ \\
\hline H-11P & 51,12 & 995,14 & 52,5 \\
\hline H-Pococí & 47,00 & 1378,75 & 64,5 \\
\hline H-11G & 43,75 & 1014,93 & 44,5 \\
\hline H-10P & 42,50 & 1412,33 & 60,6 \\
\hline H-10G & 30,50 & 1422,63 & 43,2 \\
\hline
\end{tabular}

Los híbridos de la línea progenitora $\mathrm{MHO}-10(\mathrm{H}-10 \mathrm{P}$ y H-11P) produjeron en promedio 9,69 frutos más que los híbridos con $\mathrm{MHO}-7$ ( $\mathrm{H}-10 \mathrm{G}$ y H-11G). Las plantas de los híbridos que incluyeron la línea MHO3-10 fueron más altas con respecto a las de los híbridos con MHO3-7, eso repercutió en una mayor cantidad de nudos, hojas, axilas florales y frutos por planta. También se deduce que hubo un efecto de la línea progenitora HC95-8 en la cantidad de fruta, con un promedio en sus híbridos (H-10G y H-10P) de 10,94 frutos más con respecto a los otros dos híbridos con T2 $(\mathrm{H}-11 \mathrm{G}$ y H-11P). El promedio de frutos de los cuatro híbridos fue similar a Pococí (Cuadros 4 y 5 ).

Cuadro 5. Estimados de las diferencias de número y peso promedio de frutas entre grupos de híbridos de papaya para los primeros cinco meses de cosecha. Guápiles, Limón. CR. 2009.

\begin{tabular}{|c|c|c|}
\hline Tratamientos (híbridos) & Número frutos & Peso fruto $(\mathrm{g})$ \\
\hline 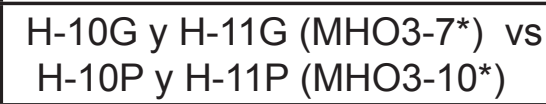 & $\begin{array}{c}-9,69 \\
(p=0,01)\end{array}$ & $\begin{array}{c}15,04 \\
(p=0,74 \text { n. s. })\end{array}$ \\
\hline $\begin{array}{l}\text { H-10G y H-10P (T2*) vs } \\
\text { H-11G y H-11P }\left(\mathrm{HC}^{*}{ }^{*}-8^{*}\right)\end{array}$ & $\begin{array}{c}-10,94 \\
(p=0,004)\end{array}$ & $\begin{array}{c}412,44 \\
(p=0,0001)\end{array}$ \\
\hline Todos vs H. Pococí & $\begin{array}{c}-5,03 \\
(p=0,21 \text { n. s. })\end{array}$ & $\begin{array}{l}-167,49 \\
(p=0,007)\end{array}$ \\
\hline
\end{tabular}

* Líneas progenitoras n. $\mathrm{s} .=$ Sin diferencias significativas. 
El peso promedio en la fruta de los cuatro híbridos fue inferior al del testigo Pococí (Cuadro $5)$. Se establecieron dos grupos de interés por el peso de la fruta (Cuadro 4), el primero, con una fruta mediana entre 1379 y $1423 \mathrm{~g}$, con potencial para el doble propósito (consumo en fresco y proceso); el segundo grupo, con fruta entre 995 y $1015 \mathrm{~g}$, un peso para el mercado de fruta pequeña, especialmente en Europa y Estados Unidos. En Cuba, Alonso et al. (2009 a), también consideran como fruta mediana la que obtuvieron en los híbridos Tainung 1 y Scarlett Princess (1456 y 1554 g). En Brasil Cattaneo et al (2007) obtuvieron un peso de fruta alrededor de los $1200 \mathrm{~g}$ en 23 genotipos del grupo Formosa así como en los dos híbridos comerciales Tainung 1 y Caliman 1 y lo consideran como un peso de fruta deseable para la comercialización.

EL promedio de producción de fruta de los híbridos $\mathrm{H}-10 \mathrm{G}$ y $\mathrm{H}-10 \mathrm{P}$, superó al de $\mathrm{H}-11 \mathrm{G}$ y $\mathrm{H}-11 \mathrm{P}$, en 412,44 gramos (Cuadro 5). Esa diferencia es un posible efecto genético de la línea T2, la cual es criolla y siempre produce una fruta grande con un peso alrededor de los 2 kg. Situación similar se ha observado en otros híbridos, en los que se han usado líneas con fruta grande $(2 \mathrm{~kg}$ ) como la Maradol roja o materiales del grupo Formosa cruzadas con materiales del grupo Solo de fruta pequeña con $500 \mathrm{~g}$ de peso (David et al. 2006).

El hibrido más productivo fue Pococí ( $p=$ 0,0004 ) con $14,3 \mathrm{~kg}$ más de fruta por planta que el promedio de los demás (Cuadro 4). También los híbridos con MHO3-10 produjeron $12,7 \mathrm{~kg}$ más $(p=0,0006)$ que los provenientes de MHO3-7 (Cuadro 4). En general los rendimientos superan la expectativa comercial que se tiene con los materiales criollos en Costa Rica, entre 31 y $37 \mathrm{~kg}$ (Bogantes y Mora 2006). Híbridos comerciales, de fruta mediana (1000-1400 g) como la Red Lady y las Tainung 1 y 2, evaluados en Puerto Rico, tuvieron rendimientos aproximados a $58 \mathrm{~kg}$ por planta (Goenaga et al. 2001). En materiales de fruta más pequeña (500-600 g) como los del grupo Solo, se obtuvieron $40 \mathrm{~kg}$ (Alonso et al. 2008 a). En el caso de Pococí, en sus pruebas iniciales se registraron rendimientos de 54,19 kg (Mora y Bogantes 2004 a).

A los ocho meses de edad, se observaron 10 plantas del híbrido H-10G y una planta en el $\mathrm{H}-11 \mathrm{G}$ con al menos una flor hermafrodita con carpelodia. Ambos descienden de la línea MHO3-7, en la cual se han detectado individuos con esta característica en la cuarta generación de autofecundación. Este fenómeno consiste en la transformación de los estambres en carpelos (Storey 1967), que es estimulada por temperaturas frías (se da a menos de $20^{\circ} \mathrm{C}$ en nuestro medio). El resultado final es una fruta deformada conocida como "cara de gato", sin valor comercial (Da Silva et al. 2006).

\section{Firmeza, brix y antracnosis}

La firmeza de cáscara y pulpa en la fruta varió entre 70 y 136 newton (N) (Cuadro 6). Pococí obtuvo $33 \mathrm{~N}$ menos de firmeza en su fruta con respecto al promedio de los cuatro híbridos restantes (Cuadro 7). Una resistencia a la penetración (firmeza) de $50 \mathrm{~N}$, utilizando extremo redondo y plano para penetrar la cáscara y pulpa, es aceptable para fruta madura que no requiere de mucha manipulación. Para fruta con mucha manipulación o que se transporta a destinos largos, se requiere de una resistencia mayor, entre 60 a $70 \mathrm{~N}$ (usando la misma técnica de penetración). No obstante, los autores han observado que una fruta $100 \%$ madura con firmeza de cáscara y pulpa por encima de $120 \mathrm{~N}$, se siente bastante dura al tacto y esa característica no satisface completamente al consumidor ni al productor; es probable que este último, confunda esa firmeza con un trastorno fisiológico que se da en algunas ocasiones y que consiste en un endurecimiento o falta de maduración de la fruta por causas aún desconocidas. 
Cuadro 6. Firmeza, brix de la pulpa y porcentaje de área de la fruta de papaya afectada por antracnosis en los híbridos, evaluados a los 10 meses. Guápiles, Limón, CR. 2009.

\begin{tabular}{|l|c|c|c|}
\hline Híbrido & Firmeza (Newton) & Grados Brix & $\%$ antracnosis \\
\hline H-11P & 136 & 12,82 & 3,54 \\
\hline H-Pococí & 70 & 10,90 & 1,00 \\
\hline H-11G & 112 & 11,71 & 5,47 \\
\hline H-10P & 74 & 11,44 & 3,67 \\
\hline H-10G & 90 & 10,51 & 7,75 \\
\hline
\end{tabular}

Tratamiento con igual letra en una misma columna presentan diferencias no significativas según Prueba de Duncan a $5 \%$.

Los híbridos derivados de HC95-8 (H-11G y H-11P) tuvieron $42 \mathrm{~N}$ más de firmeza en su fruta con respecto a (H-10G y H-10P) que se derivaron de la línea T2 (Cuadro 7). En efecto la línea HC958 es un material estable y con una fruta bastante firme (más de $100 \mathrm{~N}$ ). Fonseca et al. (2003) en una valoración de firmeza de las papaya Sunrise y Golden del grupo Solo, señalan que una baja firmeza de pulpa promueve menor resistencia al transporte y mayor cuidado para el manejo postcosecha.

Cuadro 7. Estimados de las diferencias de firmeza, brix de la pulpa y porcentaje de antracnosis entre grupos de híbridos de papaya, evaluados a los 10 meses. Guápiles, Limón, CR. 2009.

\begin{tabular}{|c|c|c|c|}
\hline Tratamientos (híbridos) & Firmeza (N) & Grados Brix & $\begin{array}{c}\text { Antracnosis } \\
(\%)\end{array}$ \\
\hline $\begin{array}{c}\mathrm{H}-10 \mathrm{G} \text { y } \mathrm{H}-11 \mathrm{G}\left(\mathrm{MHO} 3-7^{*}\right) \\
\text { vs } \\
\mathrm{H}-10 \mathrm{P} \text { y } \mathrm{H}-11 \mathrm{P}\left(\mathrm{MHO}-10^{*}\right)\end{array}$ & $\begin{array}{c}-4,00 \\
(p=0,74 \text { n. s. })\end{array}$ & $\begin{array}{c}-1,02 \\
(p=0,0003)\end{array}$ & $\begin{array}{c}-3,00 \\
(p=0,04)\end{array}$ \\
\hline $\begin{array}{c}\mathrm{H}-10 \mathrm{G} \text { y } \mathrm{H}-10 \mathrm{P}\left(\mathrm{T}^{*}\right) \\
\text { vs } \\
\mathrm{H}-11 \mathrm{G} \text { y } \mathrm{H}-11 \mathrm{P}\left(\mathrm{HC} 95-8^{*}\right)\end{array}$ & $\begin{array}{c}-42,00 \\
(p=0,0001)\end{array}$ & $\begin{array}{c}-1,30 \\
(p=0,0001)\end{array}$ & $\begin{array}{c}1,21 \\
(p=0,51 \text { n. s. })\end{array}$ \\
\hline Todos vs H. Pococí & $\begin{array}{c}-33 \\
(p=0,0003)\end{array}$ & $\begin{array}{c}-0,72 \\
(p=0,0001)\end{array}$ & $\begin{array}{c}-4,10 \\
(p=0,006)\end{array}$ \\
\hline
\end{tabular}

* Líneas progenitoras n. s.= Sin diferencias significativas.

La concentración de azúcares es una característica importante en la calidad comercial de la papaya (Mora y Bogantes 2004 b). Las frutas en los diferentes híbridos tuvieron un brix cercano 0 superior a los 11 grados (Cuadro 6). Para las variedades tipo hawaiano 11,5 grados brix, ha sido establecido como el mínimo aceptable (Paull, citado por Zhou et al. 2000) así como para la papaya de exportación (Alonso et al. 2008 b).

El híbrido Pococí resultó con 0,72 grados brix por debajo del promedio de los demás, mientras que los derivados de MHO3-10, superaron en 1,02 grados a los híbridos con MHO3-7 (Cuadro 7). Los híbridos con HC95-8 ( $\mathrm{H}-11 \mathrm{G}$ y H-11P) tuvieron fruta con 1,30 grados brix por encima de los que tuvieron T2 ( $\mathrm{H}-10 \mathrm{G}$ y H-10P) (Cuadro 7). Materiales con grados brix altos (alrededor de $12,0)$ como las líneas $\mathrm{MHO}-10$ y la $\mathrm{HC} 95-8$, son resaltados por su contribución en el aumento 
de dichos sólidos solubles en los híbridos en los que son utilizados (David et al . 2006). En México Mirafuentes y Aspeitia (2008), reportan 12,44 grados brix para un nuevo híbrido denominado Azteca, el cual compararon con la variedad Maradol, en cuya fruta obtuvieron 10,48 grados brix. Cattaneo et al. (2007), obtuvieron un brix por debajo de 10,00 grados, en frutas de 23 genotipos del grupo Formosa y en el híbrido Tainung 1, evaluados en Brasil.

La severidad de antracnosis en la fruta, en general, no llegó al $10 \%$ en ninguno de los híbridos evaluados (Cuadro 6). Es importante resaltar que solo se hizo una evaluación y además en las condiciones del productor se hacía control químico de la enfermedad.

En las condiciones en que se evaluó la antracnosis en la fruta, el híbrido Pococí tuvo un $4,10 \%$ menos severidad que el promedio de los demás híbridos (Cuadro 7). Además, se observó un 3\% menos de antracnosis en la fruta de los híbridos con MHO3-10 (H-10P y $\mathrm{H}-11 \mathrm{P})$ comparados con los promedios de los híbridos con MHO3-7 (H-10G y H-11G). La MHO3-7 es una línea que siempre ha tenido mayor incidencia de antracnosis en fruta que la línea MHO3-10, durante los procesos de selección (Cuadro 7). En papaya, esta enfermedad es la más limitante en el período poscosecha, la remoción de pecíolos como práctica cultural (Durán et al. 2000) y el control químico preventivo con daconil (Solano y Arauz 1995) son quizá las dos herramientas de combate más importantes en nuestro país; por ese motivo, la resistencia o alta tolerancia a dicha enfermedad es una de las características más importante en cualquier nueva variedad de papaya.

En términos generales los híbridos $\mathrm{H}-10 \mathrm{P}$ y Pococí, demostraron ser los más productivos y con buenas características de fruta. El híbrido $\mathrm{H}-10 \mathrm{G}$ fue el de menos rendimiento por planta y con más porcentaje de antracnosis en fruta. El híbrido $\mathrm{H}-11 \mathrm{P}$ de fruta pequeña fue productivo y el de mayor brix $(12,82)$, pero su fruta es muy dura o sea con una firmeza muy alta $(136 \mathrm{~N})$.
Agradecimiento: Los autores manifiestan su agradecimiento al técnico Felipe Rojas A. y al señor Luis Aguirre por su apoyo en las evaluaciones y el trabajo para el mantenimiento del experimento en campo.

\section{LITERATURA CITADA}

Alcántara, J.; Castro, E.; Ayvar, S.; Nava, A.; Brito, T. 2010. Características fenotípicas y agronómicas de seis genotipos de papaya (Carica papaya L.) de Tuxpan, Guerrero, México. Revista Venezolana de Ciencia y Tecnología de Alimentos. Enero-Junio 1(1):3546.

Alonso, M.; Farrés, E.; Tornet, Y.; Castro, J.; Ramos, R.; Rodríguez, M. 2008 a. Evaluación de tres cultivares de papaya del grupo Solo basada en caracteres de crecimiento y productividad. Cultivos Tropicales 29 (2):5964.

Alonso, M.; Tornet, Y.; Ramos, R.; Aranguren, M.; Rodríguez, K.; Pastor, M. 2008 b. Caracterización de los frutos decuatrocultivares de papaya del grupo Solo, introducidos en Cuba. Agronomía Costarricense 32(2):169175.

Alonso, M.; Tornet, Y.; Ramos, R.; Farrés, E.; Rodríguez, D. 2009 a. Evaluación de dos híbridos de papaya introducidos en Cuba. Agronomía Costarricense. 33(2):267-274.

Alonso, M.; Bautista, M.; Ortiz, M.; Quiroz, A.; Rohde, W.; Sánchez, L. 2009 b. Caracterización de accesiones de papaya (Carica papaya L.) a través de marcadores AFLP en Cuba. Revista Colombiana de Biotecnología Vol. XI (2):3139.

Bogantes A., Mora E. 2006. Validación del rendimiento del hibrido de papaya "Pococí" (Carica papaya). En el Caribe de Costa Rica. Revista Alcances Tecnológicos. Año 4. no. 1: 53-57.

Cattaneo, L.; Santana , A.; Gonzaga M.; Santana, E. 2007. Produtividade de cultivares 
de Mamoeiro do grupo 'Formosa' no estado do Espírito Santo. Papaya Brasil: 366-368.

Disponible en http://www.fundagres.org.br/ downloads/pi-mamao/2007melhoramento\%20 genetico_07.pdf . consultado 9 de abril del 2011.

Chan, Y.K. 1992. Progress in breeding of F1 papaya hybrids in Malaysia. Acta Hort. 292:4150.

Da Silva, F.; Gonzaga, M.; Correa, P.; Santana, T.; Pio, A.; Figueiredo, R.; Cancela, H.; Ferreguetti, G. 2007. Evaluation of the sexual expression in a segregating $\mathrm{BC} 1$ papaya population. Crop Breeding and Applied Biotechnology 7:16-23.

David M, S.; Gonzaga, M.; AmaraL, A.; Peres, A.; Ide, C. 2006. Partial diallel to evaluate the combining ability for economically important traits of papaya. Scientia Agrícola, 63(6):7p.

Durán, A.; Mora, D.; Ramírez, L. 2000. Los pecíolos de la papaya como fuente de inóculo de la antracnosis y su eliminación como práctica de control. Agronomía Mesoamericana. 11(2):07- 14.

Fonseca, M.; Rocha, N.; Cenci s.; Cecon, P.; Bressan R. 2003. Comparación entre las papayas var. Sunrise Solo y var. Golden, durante siete estados de madurez. Revista Iberoamericana de Tecnología Postcosecha. 5(2):86-91.

Goenaga, R.; Irizarry, H.; Rivera, E. 2001 Yield and fruit quality of papaya cultivars grown at two locations in Puerto Rico. J. Agric. Univ. P.R. 85 (3-4):127-134.

Guzmán D. G. 1998. Guía para el cultivo de la papaya. Ministerio de Agricultura y Ganadería. San José. Costa Rica. p. 62.

López, P. G.; López, P. J. 1995. Introducción al Micro SAS. Aplicación al análisis de experimentos. Unidad de Informática y Bioestadística. Centro Agronómico de
Investigación y Enseñanza (CATIE). Turrialba. Costa Rica. 119 pp.

Loyola, L.; Firmino De Lima, J. 2001. Selecao e Recomendacao de variedades de mamoeiro- Avaliacao de linhagens e híbridos. Revista Brasileira de Fruticultura Jaboticabal. 23(3):617-621.

Mirafuentes, F. ; Aspeitia, A. 2008. 'Azteca', primer híbrido de papaya para el trópico de México. Revista Fitotecnia México. 31(3):291293.

Mora, E., Bogantes, A 1999-2002. Evaluación del potencial comercial de líneas S2 de papaya (Carica papaya L.) en la Región Atlántica de Costa Rica. Rev. Agronomía Tropical 32: 7380.

Mora, E.; Bogantes, A. 2004 a. Evaluación de Híbridos de papaya (Carica papaya) en Pococí, Limón. Costa Rica. Agronomía Mesoamericana 15(1):39-44.

Mora, E., Bogantes, A. 2004 b. Herencia de la concentración de los sólidos solubles entre líneas parentales de papaya (Carica papayaL.) y sus híbridos. Agronomía Mesoamericana 15 (1):81-84.

Páez, A. s. f. Tecnologías sostenibles para el manejo de la antracnosis en papaya y mango. CORPOICA TURIPANÁ:http:// www.turipana. org.co/manejo_antracnosis.htm. 15 diciembre del 2010.

Rivas V, P.; Mora, G.; Téliz, D.; Mora, A. 2003. Influencia de variedades y densidades de plantación de papayo (Carica papayaL.) sobre las epidemias de mancha anular. Revista Mexicana de Fitopatología. julio-diciembre, 21 (02): 109-116.

Solano, V. ; Arauz, L. F. 1995. Combate de antracnosis en frutos de papaya mediante aplicaciones de fungicidas en el campo en la zona Atlántica de Costa Rica. Agronomía Costarricense 19 (2):25-30. 
Storey, W. B. 1967. Theory of the derivations of the unisexual flowers of Caricaceae. Agronomía Tropical 17: 273-321.

Zhou, L.; Chistopher, D.; Paull, R. 2000. Defoliation and fruit removal effects on papaya fruit production, sugar metabolism, and sucrose metabolism. J. Amer. Soc. Hort. Sci. 125 (5):644-652. 